\title{
Jury still out on cold fusion?
}

SIR - The News story "Scientists lose cold fusion libel case" and part of a leading article in the same issue (Nature 380, 369 \& 367 ; 1996) might convince readers that an Italian court has actually found a proof of misconduct by Martin Fleischmann, Stanley Pons and three Italian scientists (two of whom are the present writers).

In fact, the court's verdict, against which we shall appeal, falls short of your hasty conclusion. The court, following the conclusions of the court-consultant, acknowledges (page 13) that "the cells with electrodes of treated palladium with heavy water solutions, undoubtedly [our italics] produce an unexplainable quantity of heat", and there is no mention of fraud or misconduct anywhere in the text.

Unfortunately, it is on the widespread innuendo, defamation and vituperation that have characterized the response in scientific circles that the court bases the right of the journalist to report such defamations as "the voice of the scientific communtity" with no regard to the honour of dedicated and honest individuals. On page 13 , the Italian text reads: "One must thus reckon that the severe critiques [a clear understatement!] of the promoters of cold fusion in the articles of Pace Giovanni Maria are justified by the existence of a strong opposition in the scientific community not only to the theoretical bases of the research but also to the way in which experiments were conducted, the data were published and the conclusions on the prospects of the research programme were drawn."

One might compare such a verdict with the hypothetical verdict of a German court in the 1930 s dismissing the libel case of a Jewish group against a journalist of the Volkischer Beobachter on the grounds that antisemitism was the prevalent attitude in Germany at the time. Traces of such a doubtful ethics can be found in several leading articles in Nature about the "cold fusion affair". In particular we have in mind the question at the end of David Lindley's Commentary article (Nature 344, 375-376; 1990): "Would a measure of unrestrained mockery, even a little unqualified vituperation, have speeded cold fusion's demise?"

The problem with the enemies of cold fusion, the True Unbelievers, is that they are a bit lazy. Even Lindley acknowledges that "[a]lthough cold fusion was [note the past tense even in the spring of 1990 !], in terms of 'ordinary' physics, absurd, it was not obviously so; it contravened no fundamental laws of physics", but the leaders of the scientific community don't want to do their homework and prove their point (either experimentally or theoretically). They simply dismiss everything that supports this new scientific development and, when cornered by fact and logic, they explode in a burst of insults, while, when required to give a proof of their charges (fraud like cold fusion should in the end be proven!), they appeal to the right of "free press". It is for this reason that we will appeal against this "liberal" verdict and will continue to defend the right to "free research and discovery" in a scientific community where too many 'ayatollahs' pretend to know in advance what is right or wrong. This attitude has the obvious risk that such loud editorials, statements, judgements and the like will eventually bring an unbearable mass of ridicule upon their authors. And, judging from the positive evidence on cold fusion being accumulated, it is very likely that this misfortune will happen very soon to the most vocal among them.

\section{Emilio Del Giudice}

Isituto Nazionale di Fisica Nucleare,

Sezione di Milano,

via Celoria 16,

20133 Milano, Italy

Guiliano Preparata

Dipartimento di Fisica,

Università di Milano,

via Celoria 16,

20133 Milano, Italy

\section{The $g$ factor}

SIR - N. J. Mackintosh in his review of The $g$ Factor by Christopher Brand ${ }^{1}$, is incorrect in stating that neither Brand nor anyone else "ever established that inspection time [IT] owes its correlation with IQ scores to its correlation with $g$ ".

IT is the speed with which a person can make a simple visual (or auditory) discrimination. The $g$ factor is the largest or highest-order factor common to all of the tests in a battery of psychometric measures of cognitive abilities, such as the collection of diverse subtests typically used to obtain an IQ score $^{2}$. A factor analysis of the correlations among various cognitive tests invariably yields a general factor $(g)$ and two or more other factors that are correlated with more specific abilities, such as verbal, spatial and memory. A meta-analysis of all the reported correlations between IT and various IQ and other psychometric tests published before 1989 gave an average correlation of -0.54 after correction for artefactual sources of error $(-0.30$ before correction $)^{3}$

The question raised by Mackintosh, put more precisely, is whether IT is correlated more with the $g$ factor than it is with factors other than $g$ in test batteries used to measure IQ. There are three ways to answer this question empirically: (l) factor analyse IT among a battery of psychometric tests, (2) correlate $g$ factor scores and non- $g$ factor scores derived from the various sub- tests of an IQ battery, and (3) correlate the column vector of (a) the correlations between IT and each of the subtests, and (b) the column vector of the correlations between each of the subtests and $g$. Application of these methods shows that the $g$ factor is in fact the main source of the commonality between IT and IQ.

IT had larger correlations with $g$ than with any other psychometric factors (independent of $g$ ) in two studies ${ }^{4,5}$. Both studies also show a high correlation between the vectors of IT and $g$ subtests. In a factor analysis of the correlations between IT and eleven psychometric tests (Raven's Advanced Progressive Matrices and the ten subtests of the Multidimensional Aptitude Battery) administered to 101 college students, IT has its largest correlation with the $g$ factor, a much smaller correlation with the spatial factor and a near-zero correlation with the verbal factor, both factors independent of $g$. (The correlations used for this factor analysis, all from one study, can be found in refs. 6 and 7.)

\section{Arthur R. Jensen}

School of Education,

University of California,

Berkeley, California 94720, USA

1. Mackintosh, N. J. Nature 381, 22 (1996).

2. Jensen, A. R., \& Weng, L.-J. Intelligence 18, 231-258 (1994).

3. Kranzler, J. H. \& Jensen, A. R. Intelligence 13, 329-347 (1989).

4. Nettelbeck, T. \& Rabbitt, P. M. A. Intelligence 16, 189 205 (1992)

5. Deary, I. Intelligence 17, 223-236 (1993).

6. Kranzler, J. H. \& Jensen, A. R. Intelligence 15, 397-422 (1991).

7. Carroll, J. Intelligence 15, 423-436 1991).

\section{Last of the...}

SIR — Mark Smith (Nature 381, 272; 1996) is of course quite correct in pointing out that the last extant representative of any particular lineage may be called a relict, but unfortunately this word has the wrong connotations: it evokes more of past greatness than present catastrophe and it is more suggestive of persistence than extinction. As for the other words proposed Chaucer notwithstanding - 'endling' is a less ugly word than 'ender', but it does sound somewhat pathetic, as Elaine Andrews pointed out (Nature 381, 272; 1996). Her suggested 'terminarch', on the other hand, is certainly stronger, but is such a 'positive ring' really appropriate here? For what we hope are obvious reasons, we should like to propose the shamelessly romantic term 'mohican'; it is more poignant than pathetic and alludes straightforwardly to the tragic.

\section{Jane E. Lewis}

Paul Barlow

Institute of Marine Biology,

National Taiwan Ocean University,

2 Pei-Ning Road,

Keelung,

Taiwan, ROC 